\title{
Nach Hygieneplan gereinigt und desinfiziert
}

Multiresistente Keime, Risikopatienten, Mitarbeiterschutz und Haftungsrisiko aus Gründen wie diesen ist eine gründliche Desinfektion in der modernen zahnmedizinischen Praxis

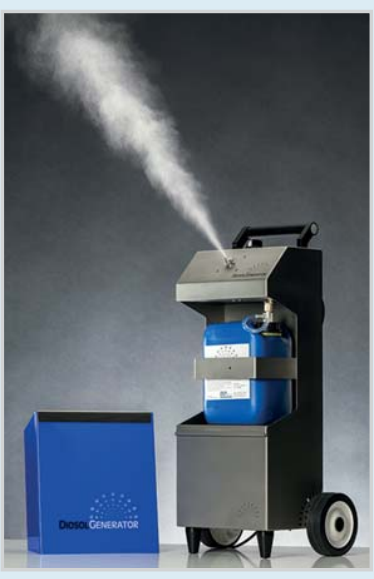

unverzichtbar. Dabei stellt es täglich eine neue Herausfordernung dar, den geforderten hohen Hygienestandard sicher zu erfüllen. Eine Möglichkeit eröffnet jetzt die Kaltvernebelungstechnologie (DioProtection, DIOP, Rosbach). Sie ergänzt die Scheuer-und-WischDesinfektion und unterstützt wirkungsvoll die gewünschte Keimreduktion und damit die Verminderung des Restrisikos in Richtung 0. Die DIOP GmbH \& Co. KG ist Experte auf dem Gebiet der Hygiene und Infektionskontrolle. Die Wurzeln seiner heutigen Expertise liegen in der Schütz Dental GmbH. Dort entwickelte man das SeptProtector-System, ein mobiles System für Raumdesinfektionen. Sein Herzstück: ein schwebefähiges Aerosol, mit dem sich die Wirksubstanzen Wasserstoffperoxid und Silber bis in die hintersten Winkel fein verteilen. Damit ergänzt das DioProtection-System wirkungsvoll die übliche Flächenhygiene. Das Konzept erfüllt die Anforderungen der BioStoffV (Biostoffverordnung) an den Arbeitsschutz sowie die Voraussetzungen für ihre Umsetzung mithilfe der TRBA 250 (Technische Regel für Biologische Arbeitsstoffe im Gesundheitswesen und in der Wohlfahrtspflege). Auch folgt das Verfahren den Empfehlungen der KRINKO und des RKI, insbesondere denjenigen zur Reinigung und Desinfektion von Flächen. Wichtig: Die Kaltvernebelung verträgt sich mit so gut wie allen Materialien, inklusive empfindlicher Elektronik. So bleibt das digitale Equipment der Praxis sicher intakt (z.B. Röntgen, Intraoralkamera, CAD/CAM-Fertigungseinheit). Weitere Informationen über das Desinfektionsssystem DioProtection gibt es im Internet unter www.diopgmbh.com. Bei speziellen Fragen steht das Unternehmen unter der Telefonnummer 06003/814-800 zur Verfügung.

Nach einer Pressemitteilung der DIOP GmbH \& Co. KG, Rosbach www.diopgmbh.com 